\section{OPEN ACCESS}

Edited and reviewed by Guohui Wan,

Sun Yat-sen University, China

*Correspondence:

Song-Mei Liu

smliu@whu.edu.cn

Wei Zhang

wei.zhang1@northwestern.edu

${ }^{t}$ These authors have contributed equally to this work

${ }^{\ddagger}$ These authors share senior authorship

Specialty section:

This article was submitted to Molecular and Cellular Oncology,

a section of the journal

Frontiers in Oncology

Received: 25 July 2021

Accepted: 13 August 2021

Published: 07 September 2021

Citation:

Li L-M, Chen C, Ran R-X,

Huang $J-T$, Sun $H-L$, Zeng $C$, Zhang Z, Zhang W and Liu S-M (2021)

Corrigendum: Loss of TARBP2 Drives the Progression of Hepatocellular Carcinoma via miR-145-SERPINE1 Axis.

Front. Oncol. 11:746958. doi: 10.3389/fonc.2021.746958

\title{
Corrigendum: Loss of TARBP2 Drives the Progression of Hepatocellular Carcinoma via miR-145-SERPINE1 Axis
}

\author{
Li-Man $\mathrm{Li}^{1 \dagger}$, Chang Chen ${ }^{2 \dagger}$, Ruo-Xi Ran ${ }^{1 \dagger}$, Jing-Tao Huang ${ }^{1,3}$, Hui-Lung Sun ${ }^{4}$, \\ Chang Zeng ${ }^{2}$, Zhou Zhang ${ }^{2}$, Wei Zhang ${ }^{2,5 *}$ and Song-Mei Liu ${ }^{1,6 * \neq}$
}

${ }^{1}$ Department of Clinical Laboratory, Center for Gene Diagnosis, and Program of Clinical Laboratory Medicine, Zhongnan Hospital of Wuhan University, Wuhan, China, ${ }^{2}$ Department of Preventive Medicine, Northwestern University Feinberg School of Medicine, Chicago, IL, United States, ${ }^{3}$ Department of Clinical Laboratory, Renmin Hospital, Wuhan University, Wuhan, China, ${ }^{4}$ Department of Chemistry and Institute for Biophysical Dynamics, Howard Hughes Medical Institute, The University of Chicago, Chicago, IL, United States, ${ }^{5}$ Institute of Precision Medicine, Jining Medical University, Jining, China, ${ }^{6}$ Hubei Province Key Laboratory of Allergy and Immunology, Wuhan, China

Keywords: HCC, TARBP2, miR-145, SERPINE1, progression

\section{A Corrigendum on}

Loss of TARBP2 Drives the Progression of Hepatocellular Carcinoma via miR-145-SERPINE1 Axis

By Li L-M, Chen C, Ran R-X, Huang J-T, Sun H-L, Zeng C, Zhang Z, Zhang W, Liu S-M (2021). Front. Oncol. 11:620912. doi: 10.3389/fonc.2021.620912

In the original article, there were mistakes in Figure $\mathbf{5}$ and Figure $\mathbf{7}$ as published. In Figure $\mathbf{5 A}$ and Figure 7A, CTL should be sh-Ctrl to keep consistence of the abbreviation throughout of the manuscript. In Figure 5B, a layer was not removed due to our less carefulness during PS operation. The corrected Figures appear below.

The authors apologize for this error and state that this does not change the scientific conclusions of the article in any way. The original article has been updated.

Publisher's Note: All claims expressed in this article are solely those of the authors and do not necessarily represent those of their affiliated organizations, or those of the publisher, the editors and the reviewers. Any product that may be evaluated in this article, or claim that may be made by its manufacturer, is not guaranteed or endorsed by the publisher.

Copyright (c) 2021 Li, Chen, Ran, Huang, Sun, Zeng, Zhang, Zhang and Liu. This is an open-access article distributed under the terms of the Creative Commons Attribution License (CC BY). The use, distribution or reproduction in other forums is permitted, provided the original author(s) and the copyright owner(s) are credited and that the original publication in this journal is cited, in accordance with accepted academic practice. No use, distribution or reproduction is permitted which does not comply with these terms. 


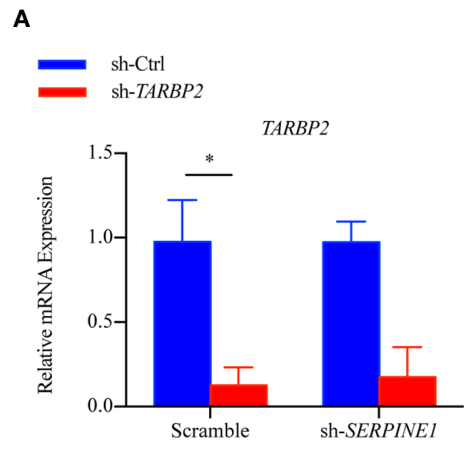

C

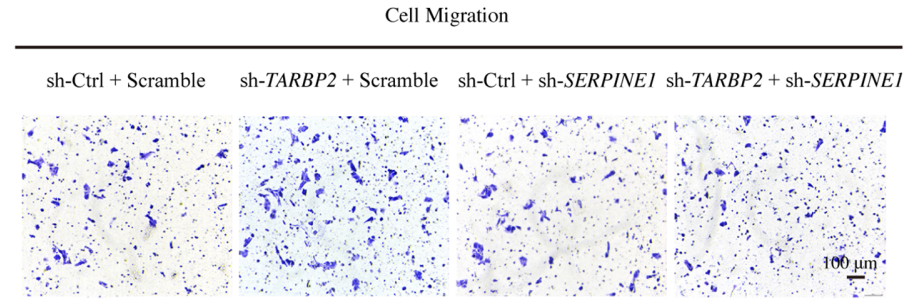

D

Cell Invasion
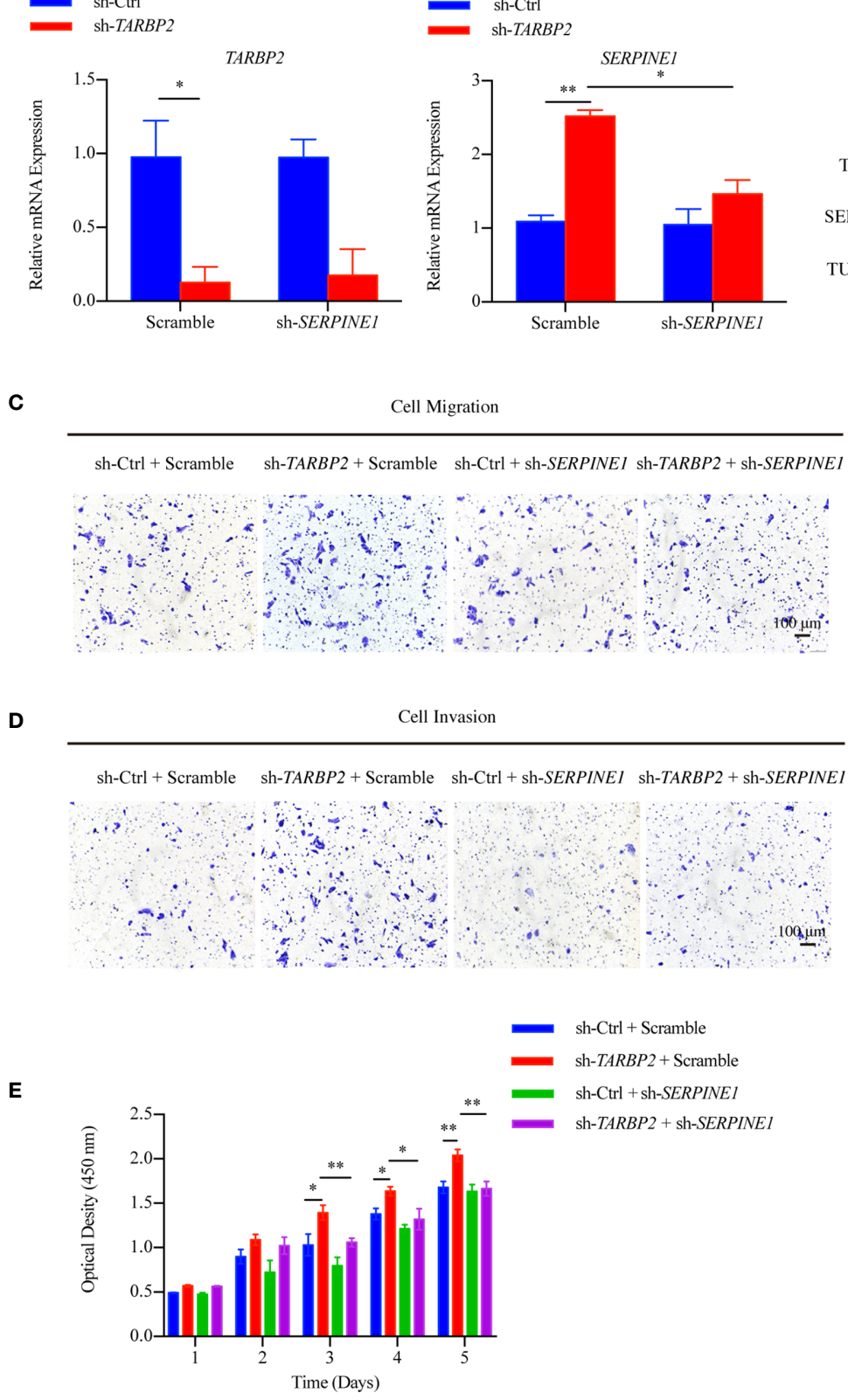

B

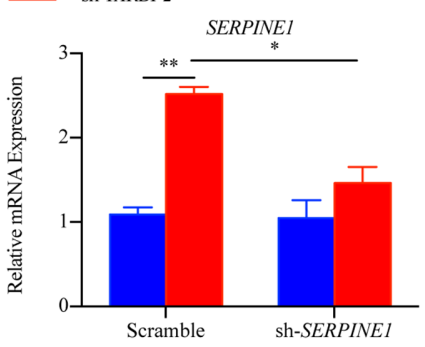

SERPINE1 (42 KDa)

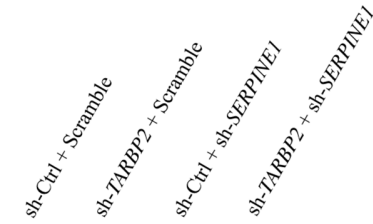

TARBP2 (42 KDa)

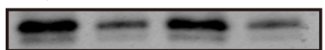

TUBULIN (52 KDa)

sh-Ctrl

sh-TARBP2

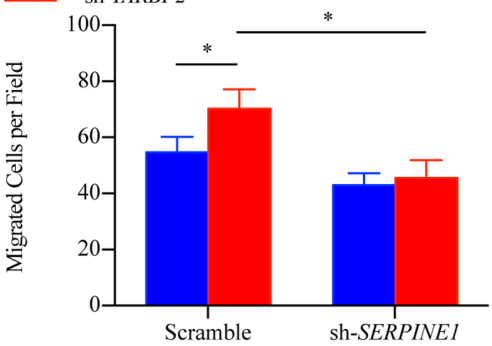

sh-Crrl

sh-TARBP2

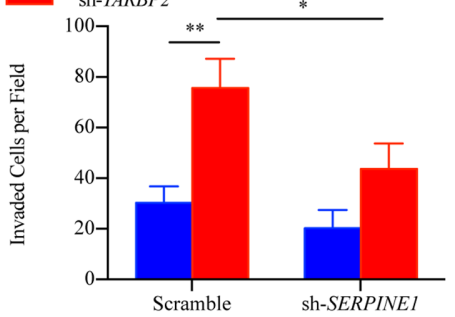

FIGURE 5 | SERPINE1 is a downstream player of TARBP2. (A, B) The mRNA and protein expression of TARBP2 and SERPINE1 in the stable sh-TARBP2 HepG2 cells after treatment with scramble control and sh-SERPINE1. (C) Cell migration; (D) Invasion; and (E) Proliferation assays of sh-TARBP2 HepG2 cells treated with scramble control and sh-SERPINE1. ${ }^{\star} p$-value $<0.05 ;{ }^{* *} p$-value $<0.01$. 
A

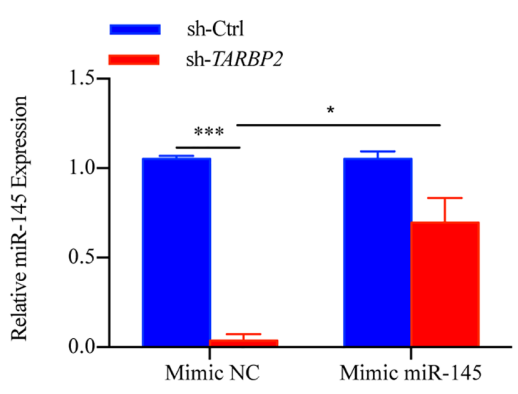

B

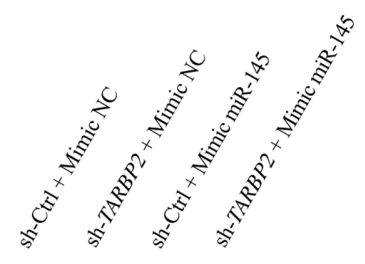

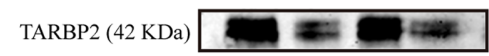

SERPINE1 (42 KDa) $\quad \mathrm{t}$

TUBULIN (52 KDa) $\longrightarrow$
C

Cell Migration

sh-Ctrl + Mimic NC sh-TARBP2 + Mimic NC sh-Ctrl + Mimic miR-145 sh-TARBP2 + Mimic miR-145

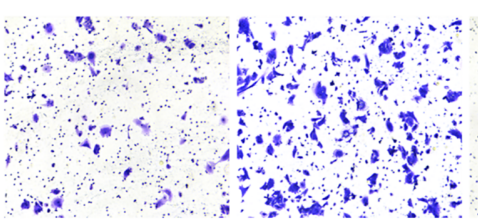

D

Cell Invasion

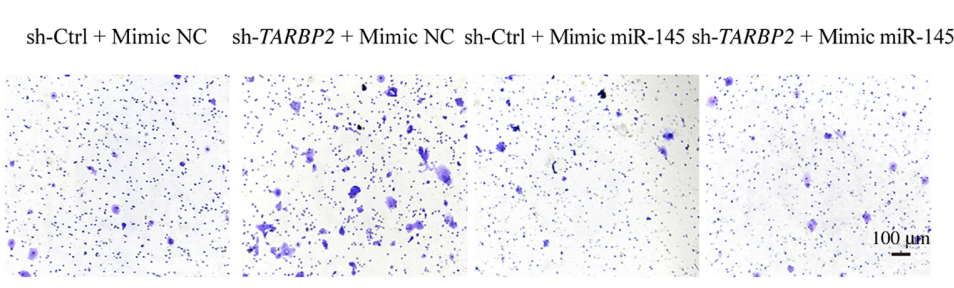

E

$$
\begin{aligned}
& \text { sh-Ctrl + Mimic NC } \\
& \text { sh-TARBP2 + Mimic NC } \\
& \text { sh-Ctrl + Mimic miR-145 }
\end{aligned}
$$$$
\text { sh-TARBP2 + Mimic miR-145 }
$$

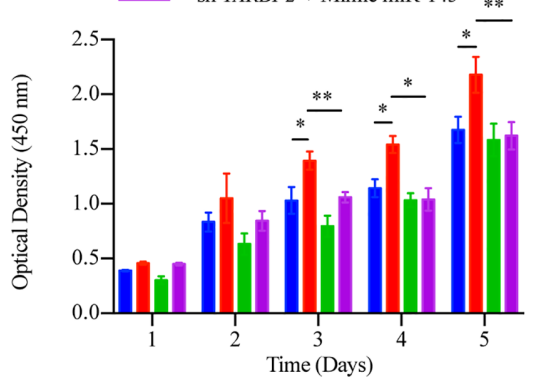

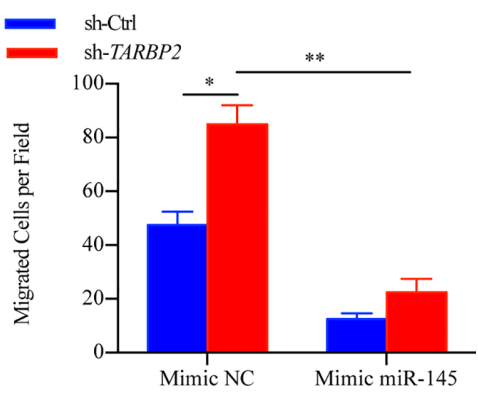

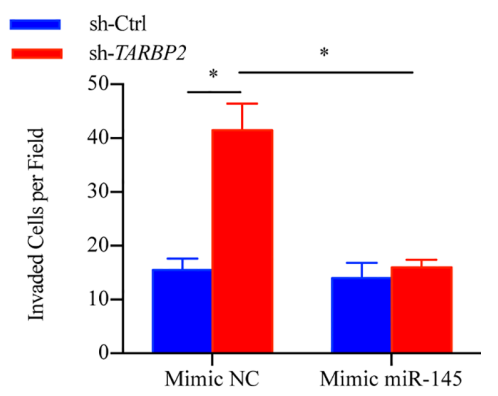

FIGURE 7 | miR-145 mediates SERPINE1 to affect the role of TARBP2 in HCC progression. (A) Levels of miR-145 in sh-TARBP2 HepG2 cells after treatment with Mimic NC and Mimic miR-145. (B) Protein levels of SERPINE1 and TARBP2 in the stable sh-TARBP2 HepG2 cells after treatment with Mimic NC and Mimic miR145. (C) Cell migration; (D) Invasion; and (E) Proliferation of sh-TARBP2 HepG2 cells treated with Mimic NC and Mimic miR-145. ${ }^{*} \mathrm{p}-\mathrm{value}<0.05$; ${ }^{* *} \mathrm{p}-\mathrm{value}<0.01$; $\mathrm{NC}$, negative control. 